\title{
Elasticity of a polydisperse hard-sphere crystal
}

\author{
Mingcheng Yang and Hongru Ma* \\ Institute of Theoretical Physics, Shanghai Jiao Tong University, \\ Shanghai 200240, People's Republic of China
}

(Dated: August 16, 2021)

\begin{abstract}
A general Monte Carlo simulation method of calculating the elastic constants of polydisperse hard-sphere colloidal crystal was developed. The elastic constants of a size polydisperse hard sphere $f c c$ crystal is calculated. The pressure and three elastic constants $\left(C_{11}, C_{12}\right.$ and $\left.C_{44}\right)$ increase significantly with the polydispersity. It was also found from extrapolation that there is a mechanical terminal polydispersity above which a $f c c$ crystal will be mechanically unstable.
\end{abstract}

PACS numbers: 05.10.Ln, 62.20.-x, 82.70.Dd

*Electronic address: hrma@sjtu.edu.cn 


\section{INTRODUCTION}

Elastic constants are among the most important physical quantities describing macroscopic mechanical behaviors of a crystal. For hard sphere crystals, the elastic constants had been calculated by various groups with different methods including density functional theory [1, 2, 3, 4, 5], Monte Carlo simulations [6, 7, 8, 9, 10 and molecular dynamics simulations [11, 12] in the last two decades. The work of Jaric [1] and Jones [2] predicted a negative poisson ratio in the hard sphere crystal, which stimulated much interest in the investigation of the elasticity of this system. However, their predictions were proved incorrect by subsequent studies [3, 4, 6, 11]. The hard sphere system is the simplest in the systems which have pure repulsion interaction, thus the study of elasticity of hard sphere crystals is an excellent starting point to the studies of more complicated and realistic repulsive systems. Due to the simplicity of the hard sphere system, it often serves as a simple model system for testing new theoretical approaches. Hard sphere model is also an important model for a large class of colloid systems, understandings of colloid systems are greatly benefited from the extensive researches of the monodisperse hard sphere model. A more realistic model describing hard sphere colloidal systems is the size polydisperse hard sphere system. The size polydispersity of colloid particles is an intrinsic property of colloidal systems [13]. The polydispersity of colloidal hard spheres is characterized by the ratio of the standard deviation to the mean of the diameter. It has a remarkable influence on the thermodynamic and dynamic behaviors of the system [14, 15, 16, 17, 18, 19, 20, 21, 22]. It is natural to expect that the elastic constants of a polydisperse hard sphere crystal differ from those of a monodisperse hard sphere crystal. However, it seems that the problem of elastic constants of polydisperse hard sphere crystals is not properly addressed in the literature to the best of our knowledge.

In this work we propose a general Monte Carlo scheme to investigate the elasticity of a polydisperse hard sphere system, by which we calculated the elastic constants of a size polydisperse hard sphere crystal. We only consider face-center-cubic $(f c c)$ crystal structure in this study, as our previous calculations [23] showed that $f c c$ structure is still the most stable one for a size polydisperse hard sphere crystal as in the monodisperse case. When simulating a polydisperse crystal the semigrand ensemble is the best candidate [15, 24, 25]. In the ensemble the imposed physical quantity is the chemical potential difference $\Delta \mu$ of particles of each kind to a reference kind. To obtain the chemical potential difference for a 
prescribed size distribution $\rho(\sigma)$, one has to solve a functional inverse problem [26, 27, 28], which can be accomplished easily by the semigrand ensemble version of the nonequilibrium potential refinement (NEPR) method (SNEPR method) [23].

Simulation approaches for calculating elastic constants of a monodisperse hard sphere crystal are divided into two categories. One is the "fluctuation" method [7] where elastic constants are related to the thermal averages of the corresponding stress components. There are some difficulties to extend the method to the polydisperse system. In the present paper we employ another method, the so called "strain" method [6, 8, 10, 11, 12], where elastic constants can be obtained from the free energy-strain relation or its first derivative, the stress-strain relation. In the simulation we used the extended ensemble method [23, 29] to determine the Helmholtz free energy of the crystal with different strain, which can also be obtained by thermodynamic integration method [30]. Then the elastic constants were extracted from the free energy-strain data.

The paper is organized as follows. In Sec. II, we introduce the model and explain how the semigrand ensemble can be applied to calculate the elastic constants of polydisperse hard sphere crystals. Section III describes the simulation method employed in this work. The computational details and results are provided in Sec. IV. Finally, we present our conclusions in Sec. V.

\section{MODEL AND THEORY}

\section{A. Semigrand canonical emsemble}

The semigrand canonical ensemble (SCE) is the most suitable ensemble for the simulation study of the elastic properties of a size polydisperse hard sphere crystal. In this ensemble the total number of particles and the distribution of particle sizes are fixed while the number of particles of each size is permitted to fluctuate. In the simulation study, the total number of particles in the system usually limited to a few hundreds to thousands, which are too small to distribute accurately according to a prescribed distribution of particle sizes. With the semigrand canonical ensemble, the distribution is realized through averages since the particle sizes are allowed to fluctuate. The grand canonical ensemble can also achieve the goal of distribution realization but insertion and deletion of particles often require more computational 
resources. For a size polydisperse system consisting of $N$ particles with the composition distribution $\rho(\sigma)$ in a constant volume $V$, the Helmholtz free energy $F(N, V,\{\rho(\sigma)\}, T)$ of the system is given by

$$
F=-P V+N \int \mu(\sigma) \rho(\sigma) d \sigma
$$

where $P$ is the pressure, $\sigma$ is the diameter of the particles, and $\mu(\sigma)$ is the chemical potential of particles with diameter $\sigma$. The semigrand canonical free energy $(\mathrm{SCFE}) Y(N, V,\{\mu(\sigma)\}, T)$ is obtained from the Helmholtz free energy by the Legendre transformation,

$$
Y=F-N \int\left(\mu(\sigma)-\mu\left(\sigma_{r}\right)\right) \rho(\sigma) d \sigma
$$

where $\sigma_{r}$ is the diameter of an arbitrarily chosen reference component. The SCFE $Y(N, V,\{\mu(\sigma)\}, T)$ is a functional of $\mu(\sigma)-\mu\left(\sigma_{r}\right)$. In semigrand canonical ensemble the thermodynamic variable to characterize the equilibrium system is $\mu(\sigma)-\mu\left(\sigma_{r}\right)$ rather than the composition distribution $\rho(\sigma)$.

The partition function $\gamma$ for this ensemble is [31]

$$
\gamma=\frac{1}{N !} \int_{\sigma_{1}} \cdots \int_{\sigma_{N}} Z_{N}\left[\prod_{i=1}^{N} \frac{1}{\Lambda^{3}\left(\sigma_{i}\right)}\right] \times \exp \left\{\beta \sum_{i=1}^{N}\left(\mu\left(\sigma_{i}\right)-\mu\left(\sigma_{r}\right)\right)\right\} \prod_{i=1}^{N} d \sigma_{i}
$$

here $\Lambda\left(\sigma_{i}\right)=h /\left(2 \pi m_{i} k T\right)^{1 / 2}$ is the thermal wavelength of the $i$ th particle and $Z_{N}$ is the canonical configuration integral for a given size configuration

$$
Z_{N}=\int_{r_{1}} \cdots \int_{r_{N}} e^{-\beta U} \prod_{i=1}^{N} d \mathbf{r}_{i}
$$

By introducing the excess chemical potential relative to ideal gas $\mu_{e x}\left(\sigma_{i}\right)=\mu\left(\sigma_{i}\right)-$ $k T \ln \left(\frac{N \Lambda\left(\sigma_{i}\right)^{3}}{V}\right)$, we can rewrite $\gamma$ in a more symmetric form

$$
\gamma=\frac{1}{N ! \Lambda^{3 N}\left(\sigma_{r}\right)} \int_{\sigma_{1}} \cdots \int_{\sigma_{N}} Z_{N} \times \exp \left\{\beta \sum_{i=1}^{N}\left(\mu_{e x}\left(\sigma_{i}\right)-\mu_{e x}\left(\sigma_{r}\right)\right)\right\} \prod_{i=1}^{N} d \sigma_{i} .
$$

The partition function $\gamma$ is related to the thermodynamic potential $Y$ through

$$
Y=-k T \ln \gamma\left(N, V, T, \mu_{e x}(\sigma)-\mu_{e x}\left(\sigma_{r}\right)\right)
$$

In practical simulations the diameter of particles are discretized into a series of special particle sizes or divide the total size range of particles into many small bins [27, 28]. As a result, the semigrand canonical partition function $\gamma$ becomes

$$
\gamma=\frac{1}{N ! \Lambda^{3 N}\left(\sigma_{r}\right)} \sum_{\sigma_{1}=\sigma_{\min }}^{\sigma_{\max }} \ldots \sum_{\sigma_{N}=\sigma_{\min }}^{\sigma_{\max }} Z_{N} \times \exp \left\{\beta \sum_{i=1}^{N}\left(\mu_{e x}\left(\sigma_{i}\right)-\mu_{e x}\left(\sigma_{r}\right)\right)\right\},
$$

here $\sigma_{\min }$ and $\sigma_{\max }$ are, respectively, the minimum and maximum of particle sizes. 


\section{B. Elastic constants of size polydisperse solid}

The elasticity of a solid is the property that the solid deforms in response to an external stress and return to its initial configuration when the stress is removed. Usually the deformation is small, otherwise the deformation may become permanent. In the case of hard sphere solids, the situation is more complicated because an external stress is necessary to stabilize the solid, while the deformation of solid is induced by exerting an excess external stress. The deformation of a continuous solid can be described by the Lagrangian strain tensor

$$
\eta_{i j}=\frac{1}{2}\left(\frac{\partial u_{i}}{\partial x_{j}}+\frac{\partial u_{j}}{\partial x_{i}}+\frac{\partial u_{k}}{\partial x_{i}} \frac{\partial u_{k}}{\partial x_{j}}\right),
$$

here $u_{i}$ is the $i$ th component of the displacement field, and $x_{i}$ is the $i$ th component of the position of the displaced point in the solid, repeated indices are summed from 1 to 3 . The Helmholtz free energy density $f=F / V$ (where $V$ is the volume of the undeformed solid) is a functional of the strain field. For a homogeneous deformation, namely the Lagrangian strain tensor $\eta_{i j}$ is independent of the position, the free energy density becomes a function of the constant strain. The elastic constants can be defined in terms of the following Taylor expansion of the free energy density

$$
f\left(\eta_{i j}\right)=f(\mathbf{0})+T_{i j}(\mathbf{0}) \eta_{i j}+\frac{1}{2} C_{i j k l} \eta_{i j} \eta_{k l}+\cdots
$$

where $f(\mathbf{0})$ is the Helmholtz free energy density of the unstrained solid, and $T_{i j}(\mathbf{0})$ is the stress tensor of the unstrained solid which is necessary to stabilize the hard sphere crystal, in the case of an $f c c$ hard sphere solid, $T_{i j}(\mathbf{0})=-p \delta_{i j}$ where $p$ is the hydrostatic pressure. The $C_{i j k l}$ is the second-order elastic constants.

It should be emphasized that in the case of a size polydisperse system, the composition distribution of the strained system must remain the same as that of the unstrained system, this is because $\eta_{i j}$ and $\rho(\sigma)$ are two independent variables of the Helmholtz free energy. Thus the explicit expression of elastic constants is

$$
C_{i j k l}=\left.\frac{\partial^{2} f(\eta)}{\partial \eta_{i j} \partial \eta_{k l}}\right|_{\rho(\sigma) ; \eta=0} .
$$

Here the subscripts $\rho(\sigma)$ means that the distribution of particle sizes is fixed during the application of strain. In semigrand canonical ensemble, the Helmholtz free energy density 
of the strained system with strain tensor $\eta$ and composition distribution $\rho(\sigma)$ is

$$
\begin{aligned}
f(\eta) & =y(\eta)+\frac{N}{V} \int\left(\mu(\sigma, \eta)-\mu\left(\sigma_{r}, \eta\right)\right) \rho(\sigma) d \sigma \\
& =y(\eta)+\frac{N}{V} \int\left(\mu_{e x}(\sigma, \eta)-\mu_{e x}\left(\sigma_{r}, \eta\right)\right) \rho(\sigma) d \sigma+\frac{3 N k T}{V} \int \ln \left(\frac{\Lambda(\sigma)}{\Lambda\left(\sigma_{r}\right)}\right) \rho(\sigma) d \sigma
\end{aligned}
$$

here $y=Y / V$. In the semigrand ensemble the composition distribution $\rho(\sigma)$ depends not only on the chemical potential difference but also on the strain $\eta_{i j}$. Therefore, in order to keep the composition distribution unchanged, the chemical potential difference $\mu_{e x}(\sigma, \eta)-\mu_{e x}\left(\sigma_{r}, \eta\right)$ has to be adjusted for each strain $\eta_{i j}$. That means at each given strain the chemical potential difference is recalculated. The evaluation of the chemical potential difference for a given composition distribution and strain can easily be performed by the SNEPR method [23], explained in the following subsection.

For the size polydisperse hard sphere $f c c$ crystal, there are only three independent secondorder elastic constants $C_{1111}, C_{1122}$ and $C_{1212}$. In the Voigt notation they can be written in a more compact format $C_{11}=C_{1111}, C_{12}=C_{1122}$ and $C_{44}=C_{1212}$. It should be noted that the elastic constants defined in this way is not the one that is measured directly in experiments, though it is widely used in theoretical investigations [5, 6, 9, 10, 11, 12].

For a cubic crystal(including $f c c$ structure) under isotropic pressure $P$ the experimentally measured elastic constants, $C^{T}$, are related to the above defined $C$ by the following relations $[4]$

$$
C_{11}^{T}=C_{11}-P ; \quad C_{12}^{T}=C_{12}+P ; \quad C_{44}^{T}=C_{44}-P .
$$

It is only when $P=0$ that the two sets of elastic constants coincide. A detail discussion of the elastic constants under stress can be found in [32].

\section{SIMULATION METHOD}

\section{A. SNEPR method}

In order to fix the composition distribution of the polydisperse crystal, we extend the NEPR algorithm [28] to the semigrand canonical ensemble. The algorithm can be used to find the chemical potential difference for a prescribed composition distribution, i.e. $\Delta \mu_{e x}(\sigma)=\Delta \mu_{e x}(\{\rho(\sigma)\})$ at a given strain. Here, we only give a brief description of the method. A detailed presentation can be found in references [23, 28]. For a given particle 

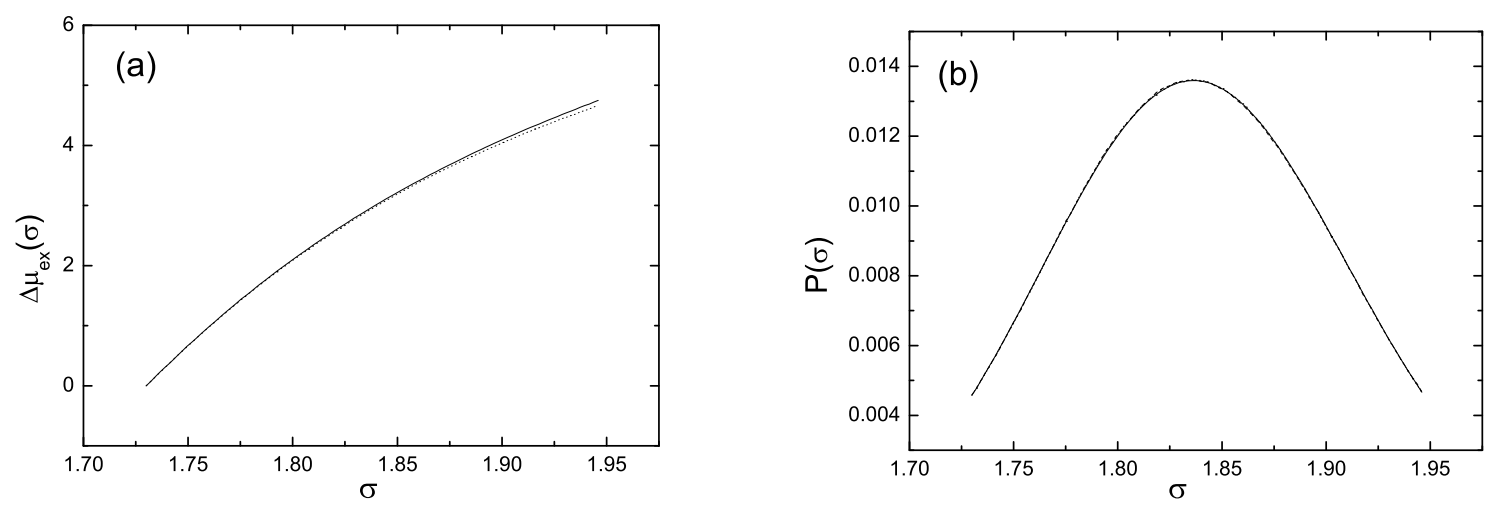

FIG. 1: (a), the solved excess chemical potential as function of the diameter of particles. Dotted line corresponds to the unstrained crystal, and solid line to the crystal with a contraction strain. (b), the solid line is the plot of the truncated Schultz function. The dotted line and dashed line are, respectively, the composition distribution for the unstrained and strained crystal, which are obtained from simulation by using the $\Delta \mu_{e x}(\sigma)$ plotted in (a). It is difficult to distinguish the three distributions.

size distribution and strain, the chemical potential difference can be calculated by a Monte Carlo iteration procedure. Firstly, initial guess of the excess chemical potential is assigned to the implement of a semigrand canonical ensemble simulation, and then it is modified at every few MC steps according to the instant size distribution $P_{i n s}(\sigma)$ as follows,

$$
\Delta \mu_{e x}^{\prime}(\sigma)=\Delta \mu_{e x}(\sigma)-\gamma_{i}\left(\frac{P_{i n s}(\sigma)-P(\sigma)}{P_{\text {ins }}(\sigma)}\right) \quad \forall \sigma .
$$

Here $\gamma_{i}$ is a modification factor of the $i$ th iteration. When the difference of the average size distribution $\bar{P}(\sigma)$ and the given composition is less than a specified value $\xi$

$$
\xi \geq \max \left(\left|\frac{\bar{P}(\sigma)-P(\sigma)}{P(\sigma)}\right|\right),
$$

one loop of the iteration is finished. The modification factor is then reduced, and the excess chemical potential of the last iteration is used as the initial input and start the next iteration. The iteration continues till the modification factor $\gamma$ reaches a very small value, typically $10^{-5}$, and the resulted excess chemical potential is then regarded as the solution of the problem.

In order to check the validity of SNEPR method, we apply it to both unstrained and strained polydisperse hard sphere crystals. Figure 1(a) are the calculated excess chemical 
potential for the truncated Schultz distribution as function of the diameter of particles. The lower dotted line and upper solid line correspond to the unstrained crystal and the crystal with a contraction strain 0.004, respectively. In the contracted crystal it is more difficult to increase the particle size, so a higher excess chemical potential is necessary to fix the composition distribution. Figure 1(b) is the comparison of the given Schultz distribution and the distribution generated with the calculated excess chemical potential. In figure 1(b) we plotted three lines of the distribution, the solid line is the given distribution, dotted line is the distribution generated from the chemical potential difference for the unstrained crystal, and dashed line is the one for the strained crystal. The agreement among the three distributions is excellent and nearly undistinguishable from the figure.

\section{B. free energy difference calculation}

To determine the elastic constants one only needs to know the Helmholtz free energy difference between the unstrained system and the strained system with the same composition distribution. The Helmholtz free energy difference can be decomposed into two parts according to equation (11)

$$
\Delta f(\eta)=\Delta y(\eta)+\Delta\left\{\frac{N}{V} \int\left(\mu_{e x}(\sigma, \eta)-\mu_{e x}\left(\sigma_{r}, \eta\right)\right) \rho(\sigma) d \sigma\right\},
$$

where $\Delta$ denotes the excess quantities relative to the unstrained system. Correspondingly, the elastic constants becomes

$$
C_{i j k l}=\left.\frac{\partial^{2}[\Delta f(\eta)]}{\partial \eta_{i j} \partial \eta_{k l}}\right|_{\rho(\sigma) ; \eta=0}
$$

The second term on the right hand side of (15) can be calculated when the $\mu_{e x}\left(\sigma_{i}, \eta\right)-$ $\mu_{e x}\left(\sigma_{r}, \eta\right)$ for the fixed composition is determined. Our main task is thus to compute the difference of semigrand free energy $\Delta y(\eta)$. To this end an extended ensemble [33] is introduced. The Lagrangian strain tensor $\eta$ is regarded as an additional ensemble variable

and different $\eta$ corresponds to different macroscopic state. The partition function of the extended ensemble is defined as

$$
\Gamma(N, T, \rho(\sigma))=\sum_{\eta=0}^{\eta_{\max }} \gamma\left(N, \eta, T, \mu_{e x}(\sigma, \eta)-\mu_{e x}\left(\sigma_{r}, \eta\right)\right)
$$


where $\eta_{\max }$ denotes the state with maximum strain and all macroscopic states $\eta$ possess the same composition distribution $\rho(\sigma)$. From equation (6) and (17) the $\Delta y(\eta)$ becomes

$$
\begin{aligned}
\Delta y(\eta) & =\ln \left[\frac{\gamma\left(N, 0, T, \mu_{e x}(\sigma, 0)-\mu_{e x}\left(\sigma_{r}, 0\right)\right)}{\gamma\left(N, \eta, T, \mu_{e x}(\sigma, \eta)-\mu_{e x}\left(\sigma_{r}, \eta\right)\right)}\right] \\
& =\ln \left[\frac{\gamma(0) / \Gamma}{\gamma(\eta) / \Gamma}\right] \\
& =\ln \left[\frac{\operatorname{Pr}(0)}{\operatorname{Pr}(\eta)}\right],
\end{aligned}
$$

where $\operatorname{Pr}(\eta)$ is the probability that the system is in the macroscopic state $\eta$. Therefore, knowledge of the macroscopic state probability distribution is sufficient to evaluate the elastic constants. The probability can be calculated from simulation by the flat histogram methods [34, 35, 36]. Here we demonstrate the implementation in the Wang-Landau scheme, other schemes can also be implemented.

The extended ensemble Monte Carlo simulation involves three kind of moves, the first is the particle displacement, the second is the particle resizing and the third is the deformation of the simulation box which corresponding to the walk in the stain $\eta$ space. The first two moves are accepted or rejected in the usual Metropolis way, i.e. if no overlap between particles happens, the trial move $(r, \sigma) \rightarrow\left(r^{\prime}, \sigma^{\prime}\right)$ is accepted with probability

$$
P_{a c c}\left(r, \sigma \rightarrow r^{\prime}, \sigma^{\prime}\right)=\min \left\{1, \exp \left(\beta \Delta \mu_{e x}\left(\sigma^{\prime}, \eta\right)-\beta \Delta \mu_{e x}(\sigma, \eta)\right)\right\}
$$

Where $\Delta \mu_{e x}(\sigma, \eta)=\mu_{e x}(\sigma, \eta)-\mu_{e x}\left(\sigma_{r}, \eta\right)$. The trial move in the $\eta$ space is treated with the Wang-Landau sampling in order to obtain the macroscopic state probability distribution $\operatorname{Pr}(\eta)$. With a initial guess of the $\operatorname{Pr}(\eta)$, the acceptance/rejection criteria for the simulation box deformation, $\eta \rightarrow \eta^{\prime}$ is

$P_{a c c}\left(\eta \rightarrow \eta^{\prime}\right)=\left\{\begin{array}{ll}\min \left\{1, \frac{\operatorname{Pr}(\eta)}{\operatorname{Pr}\left(\eta^{\prime}\right)}\left(\frac{V^{\prime}}{V}\right)^{N} e^{\beta \sum_{\sigma}\left(\Delta \mu_{e x}\left(\sigma, \eta^{\prime}\right)-\Delta \mu_{e x}(\sigma, \eta)\right)}\right\} & \text { if no overlap of spheres } \\ 0 & \text { otherwise }\end{array}\right.$,

where $\eta$ only takes some discrete values $\eta_{1}, \eta_{2} \ldots \eta_{n}$. The chemical potential difference for each strain $\eta$ were calculated with the SNEPR and stored before the simulation with extended ensemble. During the extended ensemble simulation the (unnormalized) $\operatorname{Pr}(\eta)$ is updated by multiplying a modification factor $f>1$ when a state of $\eta$ is visited, a histogram of the distribution in $\eta$ of the visited states is recorded to monitor the convergence of $\operatorname{Pr}(\eta)$. The relative probability distribution of $\eta$ is obtained at the end of the simulation, which is then 
used to determine $\Delta y(\eta)$ from equation (18). Because the composition distribution $\rho(\sigma)$ is prescribed in advance, substituting $\rho(\sigma), \Delta \mu_{e x}(\sigma, \eta)$ and $\Delta y(\eta)$ into (15) the Helmholtz free energy density difference $\Delta f(\eta)$ can then be determined. Finally, the elastic constants can be obtained from a polynomial fit to the free energy-strain data.

\section{SIMULATION DETAILS AND RESULTS}

Most of the simulations are performed with a system of 256 size polydisperse hard spheres in a parallelepiped box, periodic boundary conditions are used in all three directions. The initial configuration is an ideal $f c c$ crystal(zero strain). The density of undeformed crystal taken in this simulation is $\rho=0.576$. These value is chosen because there are some known results of the terminal polydispersity [15] and the elastic constants in the monodisperse case [12] in literature. As the previous computations [6, 9, 10, 11] the elastic constants of $f c c$ crystal can be determined completely from three independent deformations. They are the contraction, contraction-expansion and shear deformation. The composition distribution of particles used in the simulation is the truncated Schultz function

$$
P(\sigma)=\frac{1}{z !}\left(\frac{z+1}{\bar{\sigma}}\right)^{z+1} \sigma^{z} \exp \left[-\left(\frac{z+1}{\bar{\sigma}}\right) \sigma\right] \quad \sigma_{\min } \leq \sigma \leq \sigma_{\max }
$$

where $\sigma_{\min }$ and $\sigma_{\max }$ are respectively the minimum and the maximum diameters of hard sphere particles. $\bar{\sigma}$ is the average diameter and $z=\delta^{-2}-1$ controls the width of the distribution. The criteria of the truncation is that the probability density at both ends of the distribution are almost equal. They are several times less than the maximum probability density, as shown in figure 1(b). Here the probability density at $\sigma_{\min }$ and $\sigma_{\max }$ is $1 / 3$ of the peak value. In the present paper the effect of the cutoff is not studied, the emphasis is on the effect of polydispersity to the elastic properties. The size polydisperse degree is defined by $\delta=\frac{\sqrt{\overline{(\sigma-\bar{\sigma})^{2}}}}{\bar{\sigma}}$. In the simulation we consider a uniform discrete set of diameters of particles. When the number of discrete diameters is large enough, the diameter of particles tends to a continuous variable which can resemble the real polydisperse system. In this study 101 different sizes of diameters were used.

As mentioned above, the composition depends not only on the chemical potential difference but also on the strain tensor. Therefore, the chemical potential differences are calculated before the extended ensemble simulation is performed. The results indicate that 
the chemical potential difference increases with the magnitude of the strain, as plotted in Figure 1(a). In the case of a contraction deformation, the increase of the chemical potential difference is more significant than the case of shear deformation. This is reasonable because the contraction deformation consumes more configuration space so that the turn to larger sizes is more difficult and requires a larger chemical potential.

TABLE I: Elastic constants of $f c c$ hard sphere crystals and simulation parameters. All undeformed systems have the same density $\rho=0.576$. Here, $\delta$ is the size plydispersity, $N$ the number of particles and $P$ the pressure of the hard sphere crystal. The first two rows are the elastic constants of monodisperse hard sphere crystals obtained from reference [12].

\begin{tabular}{c|r|r|r|r|r}
\hline \multicolumn{1}{c|}{$N$} & $N$ & $P$ & \multicolumn{1}{c|}{$C_{11}$} & $C_{12}$ & $C_{44}$ \\
\hline 0 & 256 & & $115.5(10)$ & $32.0(4)$ & $72.4(3.2)$ \\
0 & 13292 & & $117.4(4.4)$ & $31.54(15)$ & $71.96(11)$ \\
0 & 256 & $14.54(2.5)$ & $115.1(46)$ & $32.7(16)$ & $72.0(35)$ \\
0.03 & 256 & $15.49(2)$ & $125.1(47)$ & $43.7(14)$ & $77.1(45)$ \\
0.03 & 2048 & $15.528(6)$ & $129.7(34)$ & $47.3(12)$ & $76.5(21)$ \\
0.039 & 256 & $16.24(2)$ & $135.5(33)$ & $55.9(10)$ & $78.5(45)$ \\
0.05 & 256 & $17.34(1)$ & $150.0(28)$ & $73.6(8)$ & $83.5(29)$ \\
\hline
\end{tabular}

We performed simulations for four different size polydispersity, simulation parameters and results are given in Table \. The maximum polydispersity taken in our simulation is $5 \%$, because at higher $\delta$ the crystal may be unstable [14, 15, 38]. In order to check the system size dependence we performed simulations for a larger system with the particle number $N=2048$. The difference between the two systems are clearly less than or on the same order of the statistical errors, which indicates that a system of 256 particles is already large enough to get reasonable results, similar to the monodisperse hard sphere system [10, 12]. We also calculated the elastic constants of a monodisperse hard sphere crystal using the present method, which are in full agreement with the results of Pronk and Frenkel [12]. From Table [I we see that the pressure increases with the increasing of the size polydispersity and the pressure with $\delta=0.05$ is about 20 percent higher than the monodisperse system, which is consistent with the previous reports [17, 18] that the size polydispersity can induce a higher 
osmotic pressure in the hard sphere colloidal crystal. A new phenomenon which has not been reported previously is that as the size polydispersity increases all elastic constants $\left(C_{11}\right.$, $C_{12}$ and $C_{44}$ ) of a $f c c$ hard sphere crystal increase significantly. At $\delta=0.05, C_{12}$ is 1.3 times larger than that of the monodisperse system. Figure 2 shows the ratios of $C_{11}$ to $C_{12}$ and $C_{44}$ to $C_{12}$ as a function of the polydispersity $\delta$. It is interesting to note that for the polydispersity used in the simulation these ratios nicely follow a linear relation with the polydispersity. Experimentally, Phan et al have measured the high-frequency shear modulus for the hard sphere colloidal crystals [37]. Their results are comparable to the static shear moduli of the $f c c$ crystal obtained in this study. Furthermore, our results indicate the static shear modulus also increases with the polydispersity. We think that the effect can be detected experimentally from a precise measurement.

The monodisperse hard sphere system was regarded as a representative model in the description of many different aspects of hard sphere colloids. We expected that the polydispersity may give only a small correction to the monodisperse case. The large increase of the elastic constants with polydispersity indicates that the monodisperse model has its limitations in describing the real hard sphere colloids, especially in the elastic properties. The physics behind this large increase is still not clear, one general explanation is that the elastic constants are the second derivatives of the free energy, which should be more sensitive to the polydispersity than the free energy itself. A comprehensive understanding of this enhancement of elastic constants from polydispersity requires more extensive research which is beyond the scope of this paper.

As is well known, the necessary requirements for the stability of a cubic crystal(including $f c c$ structure) are

$$
C_{11}^{T}>0 ; \quad C_{44}^{T}>0 ; \quad C_{11}^{T}+2 C_{12}^{T}>0 ; \quad\left(C_{11}^{T}\right)^{2}-\left(C_{12}^{T}\right)^{2}>0
$$

For the polydisperse hard sphere crystal under consideration, the first three conditions are obviously satisfied, the last one may be violated with increasing the polydispersity. Figure 3 depicts the change of $C_{11}^{T}-C_{12}^{T}$ with the polydispersity. The value of $C_{11}^{T}-C_{12}^{T}$ decreases with increasing polydispersity, and tends to zero at $\delta=0.0807$. To get this terminal polydispersity, we note that the simulation data follows a relation

$$
C_{11}^{T}-C_{12}^{T}=A-e^{\alpha \delta+B}
$$




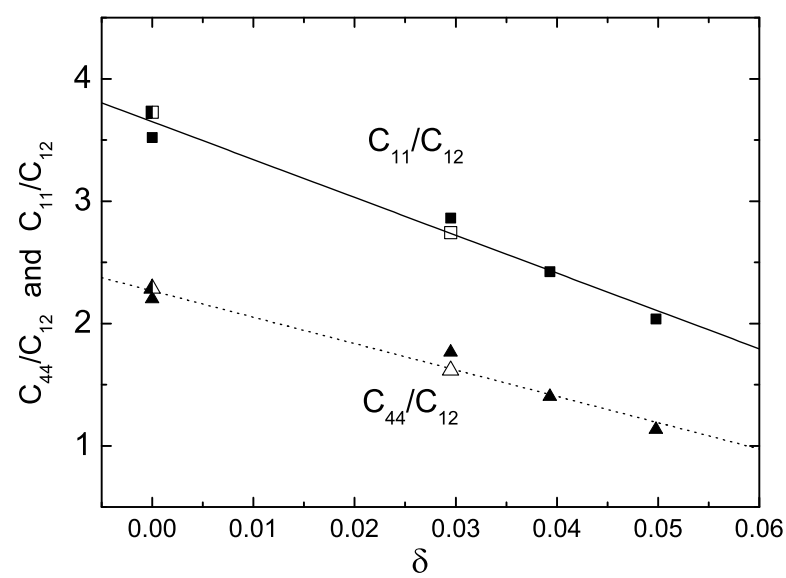

FIG. 2: The ratio of two elastic constants as a function of the size polydispersity $\delta$. The triangles and squares indicate $C_{11} / C_{12}$ and $C_{44} / C_{12}$, respectively. The filled symbols denote the data obtained from 256-particle system, open symbols from 2048-particle system. The semi-filled symbols are from a 13292-particle system given by reference [12]. The lines are linear fits to the data.

that means that $\ln \left(A-C_{11}^{T}+C_{12}^{T}\right)$ depends linearly on the polydispersity $\delta$, as shown in figure 3. Using the fitted value of $\ln A$ we can obtain the terminal polydispersity from extrapolation. This defines a mechanical terminal polydispersity (MTP) where the $f c c$ crystal becomes unstable. The strain related to the coefficient $C_{11}^{T}-C_{12}^{T}$ is the following contraction-expansion deformation:

$$
x^{\prime}=(1+\epsilon) x, \quad y^{\prime}=\frac{1}{1+\epsilon} y, \quad z^{\prime}=z .
$$

That is to say, for $\delta \geq 0.0807$ the $f c c$ crystal is no longer stable under the deformation. The instability can also be described from the point of view of soft-mode. By solving the dispersion equation, one easily finds that there exists a transverse wave, propagates along the diagonal of the (001) crystal plane and polarized in $x y$-plane, has the dispersion relation $\rho_{m} \omega^{2}=\frac{1}{2}\left(C_{11}^{T}-C_{12}^{T}\right) k^{2}$ [39], here $\rho_{m}$ is the mass density, $\omega$ is the circular frequency and $k$ the wave vector. Therefore, its frequency decreases substantially as the MTP is approached and this branch of the wave corresponds to a soft acoustical mode.

For the hard sphere crystal, it is known that there is another terminal size-polydispersity [14, 15, 16, 38] above which the crystal will not be the most stable structure, and disorder solid phase [38] or solid-solid coexistence phase [16] may become the most stable 


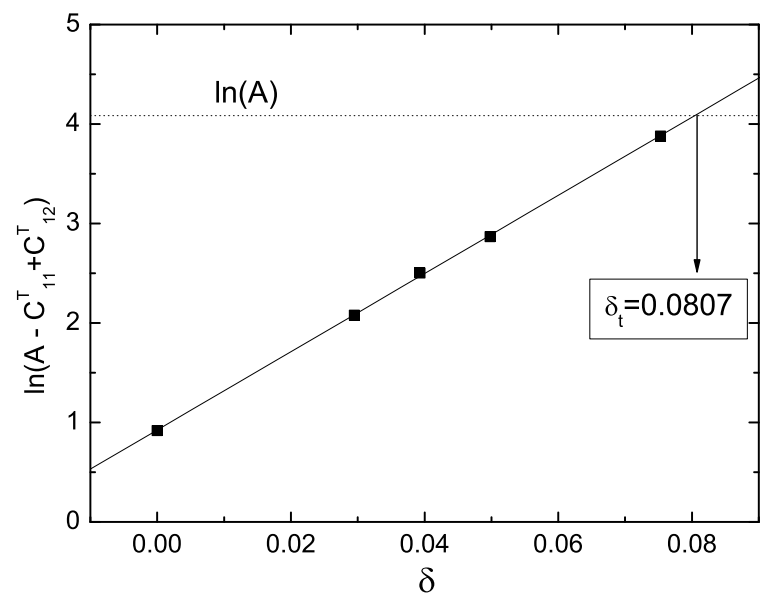

FIG. 3: The change of $C_{11}^{T}-C_{12}^{T}$ with the size polydispersity $\delta$. The solid line is a linear fit to the data. The horizontal dotted line represents the function $y=\ln (\mathrm{A})$ and $A$ is a fitted constant(see text). At $\delta=0.0807$ the solid line intersects with the dotted line.

equilibrium state. We refer to the terminal polydispersity as thermodynamical terminal polydispersity(TTP). The MTP has to be not lower than the TTP, since above TTP the $f c c$ crystal exists in a metastable state, and has good mechanical behaviors. Therefore, the MTP obtained in this study gives an upper limit of the TTP.

One important point of the semigrand canonical ensemble simulation of polydisperse hard sphere crystal is the swapping of particles. In this ensemble the particle sizes are allowed to fluctuate to get the required size distribution, with the fluctuation the particles of different sizes effectively exchange their spatial positions constantly, and with sufficient long simulation, the equilibrium state is realized during the simulation. The calculated elastic constants in this simulation is the equilibrium elastic constants, we may refer to them as ideal elastic constants. On the other hand, in real colloid crystals the particles can not exchange their positions simply because the free energy barrier is too high to be overcome in any reasonable time period. The particle arrangements are basically fixed by the process of crystal growth, which is not necessarily the equilibrium arrangement. It also noted that during the strain in the measurement of elastic constants the particles can only undergo small displacements, and it is not possible for particles to swap their positions. Based on this observation, a natural question is that wether the simulated elastic constants is the 
same one in a measurement. The answer to this question is probably yes. The reason is that measurements are always performed with a crystal of macroscopic size, which contains much more colloid particles than the number of particles in the simulation study. If the sample is well relaxed, the self-averaging effect of macroscopic number of particles may compensate the effect of particle non-swap. We expect that the ideal elastic constant can be experimentally measured after sufficient equilibration. To test the possible deviation from the ideal elastic constants, we also performed simulations in the canonical ensemble. We randomly picked up several configurations from the particle sizes distribution, and for each configuration the particle size then fixed in the subsequent simulations of applying strains and extracting elastic constants. For a system of 2048 particles we find that the elastic constants with different configuration realizations of the same distribution give elastic constants within $10 \%$ of difference. The average of the results from different realization of the configuration is listed in table II.

TABLE II: Comparison of the pressure and elastic constants obtained from the semigrand canonical ensemble simulation(upper row) and the canonical ensemble simulation(lower row).

\begin{tabular}{lcccc}
\hline & $P$ & $C_{11}$ & $C_{12}$ & $C_{44}$ \\
\hline semigrand & 15.528 & 129.7 & 47.3 & 76.5 \\
canonical & 15.591 & 137.4 & 49.9 & 75.7 \\
\hline
\end{tabular}

\section{CONCLUSION}

To conclude, the elastic constants of a $f c c$ polydisperse hard sphere crystal are simulated by the Monte Carlo method with a semigrand ensemble, the composition distribution is fixed in the simulation by the SNEPR method. The results show that both the pressure of the hard sphere solid and the three elastic constants increase with the size polydispersity $\delta$. The method can be extended to the soft sphere system and the system with other polydisperse attributes in a straightforward manner. Our results also indicate that there is a MTP where the $f c c$ crystal is unstable, which provides us an upper limit of TTP. Above TTP we do not know which of the two structure(disorder solid and solid-solid coexistence phase) is the most 
stable one. Determining the stable state from computer simulations requires more effort to accomplish.

\section{Acknowledgments}

The work is supported by the National Natural Science Foundation of China under grant No.10334020 and in part by the National Minister of Education Program for Changjiang

Scholars and Innovative Research Team in University. We thank the referee of the original manuscript to point out the possible difference when particles are not allowed to swap which is discussed in this revised one.

[1] M. V. Jaric and U. Mohanty, Phys. Rev. Lett. 58, 230 (1987); 59, 1170 (1987).

[2] G. L. Jones, Mol. Phys. 61, 455 (1987).

[3] E. Velasco and P. Tarazona, Phys. Rev. A 36, 979 (1987).

[4] H. Xu and M. Baus, Phys. Rev. A 38, 4348 (1988).

[5] B. B. Laird, J. Chem. Phys. 97, 2699 (1992).

[6] K. J. Runge and G. V. Chester, Phys. Rev. A 36, 4852 (1987).

[7] O. Farago and Y. Kantor, Phys. Rev. E 61, 2478 (2000).

[8] S. Sengupta, P. Nielaba, M. Rao and K. Binder, Phys. Rev. E 61, 1072 (2000).

[9] S. K. Kwak and D. A. Kofke, Phys. Rev. B 70, 214113 (2004).

[10] K. V. Tretiakova and K. W. Wojciechowskib, J. Chem. Phys. 123, 074509 (2005).

[11] D. Frenkel and A. J. C. Ladd, Phys. Rev. Lett. 59, 1169 (1987).

[12] S. Pronk and D. Frenkel, Phys. Rev. Lett. 90, 255501 (2003).

[13] A. v. Blaaderen and A. Vrij, Langmuir 8, 2921 (1992).

[14] P. N. Pusey and W. van Megen, Nature (London) 320, 340 (1986).

[15] P.G. Bolhuis and D. A. Kofke, Phys. Rev. E 54, 634 (1996); D. A. Kofke and P.G. Bolhuis, Phys. Rev. E 59, 618 (1999).

[16] M. Fasolo and P. Sollich, Phys. Rev. Lett. 91, 068301 (2003).

[17] S. Phan, W. B. Russel, Z. Cheng, J. Zhu, P. M. Chaikin, J. H. Dunsmuir, and R. H. Ottewill, Phys. Rev. E 54, 6633 (1996). 
[18] S. Phan, W. B. Russel, J. Zhu and P. M. Chaikin, J. Chem. Phys. 108, 9789 (1998).

[19] S. Martin, G. Bryant, and W. van Megen, Phys. Rev. E 67, 061405 (2003).

[20] R. P. A. Dullens and W. K. Kegel, Phys. Rev. Lett. 92, 195702 (2004).

[21] V. Villeneuve, R. Dullens, D. Aarts, E. Groeneveld, J. Scherff, W. Kegel and H. Lekkerkerker, Science 309, 5738 (2005).

[22] H. J. Schope, G. Bryant and W. van Megen, Phys. Rev. Lett. 96, 175701 (2006).

[23] M. C. Yang and H. R. Ma, J. Chem. Phys. 128, 134510 (2008).

[24] D. A. Kofke and E. D. Glandt, J. Chem. Phys. 87, 4881 (1987).

[25] M. A. Bates and D. Frenkel, J. Chem. Phys. 109,6193 (1998).

[26] F. Escobedo, J. Chem. Phys. 115, 5642 (2001); 115, 5653 (2001).

[27] N. B. Wilding and P. Sollich, J. Chem. Phys. 116, 7116 (2002).

[28] N. B. Wilding, J. Chem. Phys. 119, 12163 (2003).

[29] M. C. Yang and H. R. Ma, (unpublished).

[30] D. Frenkel and B. Smit, Understanding Molecular Simulation (Academic, San Diego,1996).

[31] J. G. Briano and E. D. Glandt, J. Chem. Phys. 80, 3336 (1984).

[32] T. H. K. Barron and M. L. Klein, Proc. Phys. Soc. 85, 523 (1965).

[33] A. P. Lyubartsev, A. A. Martsinovski, S. V. Shevkunov, and P. N. Vorontsov-Velyaminov, J. Chem. Phys. 96,1776 (1992).

[34] B. A. Berg and T. Neuhaus, Phys. Rev. Lett. 68, 9 (1992).

[35] F. Wang, D. P. Landau, Phys. Rev. Lett. 86, 2050 (2001); Phys. Rev. E 64, 056101 (2001).

[36] J. S. Wang and R. H. Swendsen, J. Stat. Phys. 106, 245 (2002).

[37] S. Phan, M. Li, W. Russel, J. Zhu, P. Chaikin and C. T. Lant, Phys. Rev. E60, 1988 (1999).

[38] P. Chaudhuri, S. Karmakar, C. Dasgupta, H. R. Krishnamurthy, and A. K. Sood, Phys. Rev. Lett. 95, 248301 (2005).

[39] L. D. Landau and E. M. Lifshitz, Theory of Elasticity (Beijing World Publishing Corporation, Beijing, 1999). 